\title{
THE SYLOW $p$-SUBGROUPS OF SEMICOMPLETE NILPOTENT GROUPS
}

\author{
MARTYN R. DIXON AND E. MYRON RIGSBY
}

(Communicated by Ronald M. Solomon)

\begin{abstract}
A nilpotent group whose group of outer automorphisms is trivial may contain elements of finite order. This paper is concerned with how large the Sylow $p$-subgroups of such a group can be. We show that in many cases the Sylow $p$-subgroups of such a semicomplete nilpotent group are always finite.
\end{abstract}

\section{INTRODUCTION}

The relation between the structure of a group $G$ and that of its automorphism group Aut $G$ has been the object of considerable research. Of particular interest in recent years are those groups which are semicomplete. A group $G$ is said to be complete if it has trivial center and Aut $G=\operatorname{Inn} G$. A group $G$ is said to be semicomplete if Aut $G=\operatorname{Inn} G$. Abelian groups of order greater than two are never semicomplete. Interest in automorphisms of nilpotent groups was spurred some years ago by a conjecture of Haimo and Schenkman that a nilpotent group could never be semicomplete. Results of Gaschütz [7, 8] and Zalesskii [14] combined to verify the conjecture for periodic nilpotent groups. Shortly thereafter, however, Zalesskii [15] constructed an example of a torsionfree nilpotent group that was semicomplete. Subsequent examples were provided by Heineken [9] and Robinson [10]. These groups were all torsionfree. Semicomplete nilpotent groups were further studied in [1-4]. In [3] semicomplete mixed nilpotent groups of class 2 were constructed.

Fournelle began the investigation of mixed semicomplete nilpotent groups in [2] by considering the special case in which the central quotient $G / Z(G)$ is torsionfree. He found in this case that either $G$ is torsionfree or $G=H \times C_{2}$ where $H$ is a torsionfree 2-radicable semicomplete nilpotent group and $C_{n}$ is the cyclic group of order $n$. (Here we call a group $G$ p-radicable if given $x \in G$ there exists $y \in G$ such that $y^{p}=x$. Then $G$ is radicable if and only if $G$ is $p$-radicable for all primes $p$. If $G$ is also abelian we use the term divisible rather than radicable.) The aim of this paper is to continue this investigation. In

Received by the editors December 20, 1991.

1991 Mathematics Subject Classification. Primary 20E36, 20 F28.

Key words and phrases. Automorphism, semicomplete nilpotent group.

The second author acknowledges the assistance of a Graduate Council Research Fellowship from the University of Alabama, which he received during a portion of the time this research was undertaken. 
particular we shall be concerned with the following problem, posed by Fournelle: If $G$ is semicomplete, are its Sylow $p$-subgroups necessarily finite? We have been unable to solve this problem completely, there being one case which seems to be particularly difficult to handle. Let $Z$ denote the center of $G$ and let $Q=G / Z$. Our main theorem is the following:

Theorem A. Let $G$ be a semicomplete nilpotent group and $p$ a prime. If one of the following conditions holds then the Sylow p-subgroup of $G$ is finite.

(i) $Q / Q^{\prime} Q^{p}$ is finite.

(ii) $Q_{p}$, the Sylow p-subgroup of $Q$, is countable.

(iii) $Q_{p}$ has finite exponent.

(iv) $G$ is nilpotent of class 2 .

This result extends certain results of [3] and the main result of [2]. The reader should also compare this result with [5, Theorem A] and note that Fournelle [3] has constructed uncountable semicomplete nilpotent groups. Furthermore, as far as we are aware, no examples of semicomplete nilpotent groups of class 3 or more have yet been constructed. Such examples would be of some interest. Our result cannot be strengthened further since Fournelle [3] gives examples where the torsion subgroup is infinite.

Part (i) of Theorem A takes on added significance when contrasted with the following result, which we shall also prove.

Theorem B. Let $G$ be a nilpotent group with central quotient $Q=G / Z$. If $Q / Q^{\prime} Q^{p}$ is uncountable for some prime $p$ then $G$ is not semicomplete.

Thus if $G$ is semicomplete, $\left|Q / Q^{\prime} Q^{p}\right| \leq \aleph_{0}$ for all primes $p$.

The layout of the paper is as follows. In $\S 2$ we prove the key result that the Sylow $p$-subgroup of the center of a semicomplete nilpotent group is reduced. In $\S 3$ we show that, if $\pi(G)$ denotes the set of primes $p$ for which $G$ possesses an element of order $p$, then the set $\pi(G)-\pi(G / Z(G))$ is somewhat restricted. Section 4 contains the proof of Theorem B. In $\S 5$ we prove Theorem A.

We make frequent use of a number of facts which are particularly useful when applied to nilpotent groups. For a group $N$ we denote the terms of its upper central series by $Z_{i}(N)$ or simply by $Z_{i}$, if $i \geq 0$, and then there is a monomorphism

$$
Z_{i+2}(N) / Z_{i+1}(N) \rightarrow \operatorname{Hom}\left(N_{\mathrm{ab}}, Z_{i+1}(N) / Z_{i}(N)\right) .
$$

Here $N_{\mathrm{ab}}$ denotes the abelianization of $N$. We denote the terms of the lower central series of $N$ by $\gamma_{i}(N)$, so there is an epimorphism

$$
N_{\mathrm{ab}} \otimes\left(\gamma_{i}(N) / \gamma_{i+1}(N)\right) \rightarrow \gamma_{i+1}(N) / \gamma_{i+2}(N) \text { for all } i .
$$

The reader is referred to [11] and [13] for further details of these maps. We shall also use the fact that if $A$ is a central subgroup of the group $N$ then we can identify $\operatorname{Hom}\left((N / A)_{\mathrm{ab}}, A\right)$ with the subgroup of Aut $N$ which acts trivially on $A$ and $N / A$. We also require a large number of facts concerning abelian groups, particularly properties of the functors Hom and $\otimes$, all of which can be found in Fuchs [6, Volume 1]. Our notation, when not explained, is standard. We shall denote the Sylow $p$-subgroup of a group $G$ by $G_{p}$. 


\section{2. $Z_{p}$ IS REDUCED}

We begin with the following elementary result which is no doubt well known, so we omit its proof.

2.1. Lemma. Let $p$ be a prime and $\mathfrak{m}_{1}, \mathfrak{m}_{2}, \ldots$ be a sequence of cardinals such that $\mathfrak{m}_{n} \neq 0$ for infinitely many $n$. Then $\prod_{n=1}^{\infty} \prod_{\mathfrak{m}_{n}} C_{p^{n}}$ has infinite torsionfree rank.

It is important to see how properties of the abelianization of a nilpotent group affect the whole group. Our next lemma shows how abelian subgroups are affected. We let $r_{0}(G)$ and $r_{p}(G)$ denote the torsionfree rank and $p$-rank respectively of the abelian group $G$.

2.2. Lemma. Let $N$ be a nilpotent group of class $c$. Let $A$ be an abelian subgroup of $N$ and let $r=r_{0}\left(N_{\mathrm{ab}}\right)$. Then $r_{0}(A) \leq r+r^{2}+\cdots+r^{c}$.

Proof. Let $N_{i}$, for $i=1, \ldots, c+1$, be the terms of the lower central series. It follows from our remarks in the introduction that $N_{i} / N_{i+1}$ is of torsionfree rank at most $r^{i}$. If we set $A_{i}=A \cap N_{i}$ then $A_{i} / A_{i+1}$ has torsionfree rank at most $r^{i}$ and hence $A$ has torsionfree rank as claimed in the lemma.

We shall require a theorem of Fuchs concerning the structure of $\operatorname{Hom}\left(A, C_{p^{\infty}}\right)$ for abelian groups $A$, which may be found in [6, Theorem 47.1]. For ease of reference we record this here.

2.3. Theorem. Let $p$ be a prime. Let $A$ be an abelian group and let $B$ be a $p$ basic subgroup of $A$ where $B=\bigoplus_{n=0}^{\infty} B_{n}$ with $B_{0} \cong \bigoplus_{\mathfrak{m}_{0}} \mathbb{Z}$ and $B_{n} \cong \bigoplus_{\mathfrak{m}_{n}} C_{p^{n}}$ for $n \geq 1$. Also let $\mathfrak{m}=r_{p}(A / B)$ and let $\mathfrak{t}=r_{0}(A / B)$. Then

$$
\operatorname{Hom}\left(A, C_{p^{\infty}}\right) \cong \prod_{\mathfrak{m}_{0}} C_{p^{\infty}} \oplus \prod_{n=1}^{\infty} \prod_{\mathfrak{m}_{n}} C_{p^{n}} \oplus \prod_{\mathfrak{m}} J_{p} \oplus \prod_{\mathfrak{t} \aleph_{0}} \mathbb{Q}
$$

(where $J_{p}$ is the ring of $p$-adic integers and $\mathbb{Q}$ is the additive group of rational numbers).

We use this result and its notation in the following lemma.

2.4. Lemma. Let $G$ be a semicomplete nilpotent group and suppose that $p$ is a prime. Suppose $C_{p \infty} \leq Z_{p}$, the p-component of $Z(G)$. Then

(i) $Q_{\mathrm{ab}}$ has finite torsionfree rank.

(ii) The p-component of $Q_{\mathrm{ab}}$ is bounded.

(iii) If $B$ is a p-basic subgroup of $Q_{\mathrm{ab}}$ then $Q_{\mathrm{ab}} / B$ is a $p^{\prime}$-group.

Proof. The hypothesis $C_{p^{\infty}} \leq Z_{p}$ implies $H=\operatorname{Hom}\left(Q_{\mathrm{ab}}, C_{p^{\infty}}\right)$ is a direct summand of $\operatorname{Hom}\left(Q_{\mathrm{ab}}, Z\right) \cong Z(Q)$. The structure of $H$ is given by 2.3, with $B$ p-basic in $Q_{\mathrm{ab}}$.

Note that $r_{0}\left(Q_{\mathrm{ab}}\right)=\mathfrak{m}_{0}+\mathfrak{t}$. If $\mathfrak{t}$ is infinite and $\mathfrak{t} \geq \mathfrak{m}_{0}$, then $r_{0}(H) \leq \mathfrak{t}$ by 2.2. On the other hand, $\prod_{t \aleph_{0}} \mathbb{Q} \leq H$, so $H$ has torsionfree rank at least $2^{\mathfrak{t}}$, a contradiction. If $\mathfrak{m}_{0}$ is infinite and $\mathfrak{m}_{0} \geq \mathfrak{t}$ then $r_{0}(H) \leq \mathfrak{m}_{0}$. Also,

$$
r_{0}(H) \geq r_{0}\left(\prod_{\mathfrak{m}_{0}} C_{p^{\infty}}\right)=r_{0}\left(\bigoplus_{2^{\mathrm{m}_{0}}}\left(C_{p^{\infty}} \bigoplus \mathbb{Q}\right)\right) \geq 2^{\mathrm{m}_{0}},
$$


by [6, p. 105], again a contradiction. Hence $\mathfrak{t}$ and $\mathfrak{m}_{0}$ are both finite. Thus $r_{0}\left(Q_{\mathrm{ab}}\right)$ and $r_{0}(H)$ are finite. However, $\prod_{\mathrm{t} \aleph_{0}} \mathbb{Q} \leq H$, so $\mathfrak{t}=0$. Hence $Q_{\mathrm{ab}} / B$ is periodic. Furthermore $\mathfrak{m}=0$ since $\prod_{\mathrm{m}} J_{p} \leq H$ and $J_{p}$ has infinite torsionfree rank. Hence $Q_{\mathrm{ab}} / B$ is a $p^{\prime}$-group.

By $2.1, r_{0}(H)$ finite also implies $\mathfrak{m}_{n} \neq 0$ for only finitely many $n$. Thus the torsion subgroup of $B$ has exponent equal to $p^{k}$ where $k$ is the largest value of $n$ for which $\mathfrak{m}_{n} \neq 0$. Since $Q_{\mathrm{ab}} / B$ is a $p^{\prime}$-group it then follows that $\left(Q_{\mathrm{ab}}\right)_{p}$ is the Sylow $p$-subgroup of $B$ and thus $\left(Q_{\mathrm{ab}}\right)_{p}$ is bounded. This completes the proof.

At this stage we need the following result.

2.5. Lemma. Suppose that $A$ is an abelian group and $p$ is a prime. Suppose:

(i) $A$ has finite torsionfree rank.

(ii) $A_{p}$ is bounded.

(iii) $B$ is a p-basic subgroup and $A / B$ is a $p^{\prime}$-group.

Then $A$ has no images with unbounded p-component.

Proof. By (ii) and the purity of $A_{p}$, it follows that $A=A_{p} \oplus K$, for some subgroup $K$. We claim that $K$ has no infinite $p$-groups as quotients from which the result follows immediately. Suppose that $K / R$ is an infinite $p$-group for some $R$ and let $L$ be of maximal torsionfree rank in a $p$-basic subgroup of $K$. Then $(L+R) / R$ is a $p$-group so $L / L \cap R$ is finite and we may assume $L \leq R$. Then $K / R$ is a $p^{\prime}$-group by (iii), whence $K=R$, a contradiction.

For future reference we state the following fact, the proof of which is straightforward.

2.6. Lemma. Suppose that $A$ is an abelian group and $C \leq A$. Suppose every image of $C$ and every image of $A / C$ has bounded p-component. Then every image of $A$ has bounded p-component.

We can now prove:

2.7. Lemma. Suppose $A$ and $C$ are abelian groups and $p$ is a prime. Suppose also that all images of $A$ and $C$ have bounded p-component and if $L$ is a p-basic subgroup of $C$ then $C / L$ is a $p^{\prime}$-group. Then all images of $A \otimes C$ have bounded p-component.

Proof. By hypothesis, $A$ and $C$ must themselves have bounded $p$-component and also $p$-basic subgroups of $A$ and $C$ must have finite torsionfree rank. Since $A_{p}$ and $C_{p}$ are pure bounded subgroups of $A$ and $C$ respectively, we have $A=A_{p} \oplus M$ and $C=C_{p} \oplus N$, say, where $M$ and $N$ are $p$-torsionfree. Then

$$
A \otimes C=\left(A_{p} \otimes C_{p}\right) \oplus\left(A_{p} \otimes N\right) \oplus\left(M \otimes C_{p}\right) \oplus(M \otimes N) .
$$

Since $A_{p}$ and $C_{p}$ are bounded $p$-groups, $A_{p} \otimes C_{p}, A_{p} \otimes N$, and $M \otimes C_{p}$ are also bounded $p$-groups and thus these three summands have no unbounded $p$-images.

Let $B$ and $D$ be $p$-basic subgroups of $M$ and $N$ respectively. By [6, Theorem 60.3], the exact sequences $0 \rightarrow B \rightarrow M \rightarrow M / B \rightarrow 0$ and $0 \rightarrow D \rightarrow$ $N \rightarrow N / D \rightarrow 0$ yield an exact sequence

$$
(B \otimes N) \oplus(D \otimes M) \rightarrow M \otimes N \rightarrow M / B \otimes N / D \rightarrow 0 .
$$


Now $N / D \cong C / L$ is a $p^{\prime}$-group and hence $M / B \otimes N / D$ is a $p^{\prime}$-group and consequently only has $p^{\prime}$ images. Since $B$ and $D$ have finite ranks, say $k$ and $l$ respectively, it follows that $(B \otimes N) \oplus(D \otimes M)=\left(\bigoplus_{i=1}^{k} N\right) \oplus\left(\bigoplus_{i=1}^{l} M\right)$. The hypotheses now imply that $(B \otimes N) \oplus(D \otimes M)$ has no quotients with unbounded $p$-component. The conclusion of the lemma now follows from the exact sequence (2), Lemma 2.6, and the direct sum decomposition (1).

We use these technical results to establish the following lemma.

2.8. Lemma. Let $G$ be a semicomplete nilpotent group and suppose $p$ is a prime. Suppose that $C_{p \infty} \leq Z_{p}$. Then each lower central factor $\gamma_{i} Q / \gamma_{i+1} Q$ of $Q$ has bounded p-component.

Proof. The results 2.4 and 2.5 establish that $Q_{\mathrm{ab}}$ satisfies the hypotheses for both $A$ and $C$ in 2.7. Repeated application of 2.7 yields that $\otimes^{i} Q_{\mathrm{ab}}$ has no images with unbounded $p$-component. The result then follows using the epimorphisms mentioned in the introduction.

We may now prove the main result of this section.

2.9. Proposition. Suppose that $G$ is a semicomplete nilpotent group and that $p$ is a prime. Then the Sylow p-subgroup of the center of $G$ is reduced.

Proof. Suppose that $Z_{p}$ contains a $C_{p^{\infty}}$. It follows from 2.1, 2.3, and 2.4 that the subgroup $H=\operatorname{Hom}\left(Q_{\mathrm{ab}}, C_{p^{\infty}}\right)$ is simply

$$
H=\bigoplus_{\mathbf{m}_{0}} C_{p^{\infty}} \oplus\left(\bigoplus_{n=1}^{k} \prod_{\mathbf{m}_{n}} C_{p^{n}}\right)
$$

with $\mathfrak{m}_{0}=r_{0}(B)=r_{0}\left(Q_{\mathrm{ab}}\right)$. Let $D$ be the maximal divisible subgroup of $H$ so $D \cong \bigoplus_{\mathrm{m}_{0}} C_{p^{\infty}}$, and define $D_{i}=\gamma_{i} Q \cap D$. Then, by 2.8 , the $p$-group $D_{i} / D_{i+1}$ is of bounded exponent for all $i$. Hence $\mathfrak{m}_{0}=0$. Therefore $Q_{\mathrm{ab}}$, and hence $Q \cong$ Aut $G$, is periodic. A theorem of Robinson [12, Theorem B] may now be applied to show that the torsion subgroup of $G$ has finite exponent, which contradicts our assumption. Hence $Z_{p}$ is reduced, as required.

The following result is easily established using [11, Lemma 9.35].

2.10. Corollary. If $G$ is a semicomplete nilpotent group then $\left(Z_{i+1} / Z_{i}\right)_{p}$ is reduced for all $i$.

It is now easy to establish the next result.

2.11. Corollary. If $G$ is a semicomplete nilpotent group then $(G / Z)_{p}$ contains no radicable subgroups.

\section{THE CASE $p \in \pi(G)-\pi(G / Z)$}

We begin this section with the following lemma, the proof of which we omit since it is essentially that of Lemma 3.1 in [2].

3.1. Lemma. Suppose that $G$ is a nilpotent group and $Z(G)$ contains an element of order $p$, for some prime $p$. Suppose that $p \notin \pi(\operatorname{Aut} G)$. Then either

(i) $G$ is p-radicable, or

(ii) $G=C_{p} \times H$ for some p-radicable group $H$. 
This has the following corollary.

3.2. Corollary. If $G$ is a semicomplete nilpotent group and $p \in \pi(G)-\pi(G / Z)$ then either

(i) $G$ is p-radicable, or

(ii) $p=2$ and $G=C_{2} \times H$ for some semicomplete 2-radicable group $H$.

Note that in the second case $Z(G)=Z(H) \times C_{2}$ and thus $Q=G / Z(G) \cong$ $H / Z(H)$, so $H / Z(H)$ is 2-torsionfree. We can now use the results of $\S 2$ to obtain the following:

3.3. Proposition. Suppose that $G$ is a semicomplete nilpotent group and that $p$ is a prime in $\pi(G)-\pi(G / Z)$. Then either $G_{p}$ is trivial or $p=2$ and $G_{p}$ is cyclic of order 2.

Proof. It follows from 3.2 that either $G$ is $p$-radicable or $p=2$ and $G=$ $C_{2} \times H$, for some 2-radicable group $H$. In either case $Q=G / Z$ is $p$-radicable. In the first case, if $x \in Z_{p}(G)$ then there exists $y \in G$ such that $y^{p}=x$. Hence $y$ is a $p$-element of $Z$ since $p \notin \pi(G / Z)$. Therefore, $y \in Z_{p}(G)$. A similar argument holds in the second case also. Hence either $Z_{p}(G)$ or $Z_{p}(H)$ is divisible. The result now follows from 2.9.

\section{4. $Q / Q^{\prime} Q^{p}$ UNCOUNTABLE}

In the remainder of this paper we shall assume that $p \in \pi(G / Z)$. In [14] Zalesskii defines a basic subgroup $B$ of a nilpotent $p$-group $N$ as the preimage of a basic subgroup of $N_{\mathrm{ab}}$. Such basic subgroups have several properties which we shall exploit in this section. We shall show that for a semicomplete nilpotent group the size of $Q / Q^{\prime} Q^{p}$ is somewhat restricted. First we prove

4.1. Lemma. Suppose that $N$ is a nilpotent group and $p$ is a prime. If $\left|N / N^{\prime} N^{p}\right|$ is infinite then $\left|N / N^{p^{n}}\right|=\left|N / N^{\prime} N^{p}\right|$ for all natural numbers $n$.

Proof. It follows from the epimorphisms mentioned in the introduction that $|N|=\left|N / N^{\prime}\right|$, so it suffices to prove the result for abelian groups $N$. However, if $B$ is a $p$-basic subgroup of $N$ then $N / N^{p^{n}} \cong B / B^{p^{n}}$ for all $n$, by [6, p. $144(B)]$. However, in this case, $|B|=\left|B / B^{p^{n}}\right|$ for all $n$. Hence the result.

The key result in this section is the following. It is a generalization of [6, Corollary 34.4].

4.2. Proposition. Suppose that $N$ is a reduced nilpotent p-group for some prime $p$.

(i) If $N / N^{\prime} N^{p}$ is finite then $N$ is finite.

(ii) If $\left|N / N^{\prime} N^{p}\right|=\kappa$ is infinite then $\kappa \leq|N| \leq \kappa^{\aleph_{0}}$.

Proof. (i) follows from [13, Lemma 6.10].

(ii) Suppose that $N$ has class $c$. We inductively construct subgroups $R_{i}, D_{i}$ of $N$ such that

(1) $D_{i-1} / D_{i-1}^{\prime}=R_{i} / D_{i-1}^{\prime} \oplus D_{i} / D_{i-1}^{\prime}$.

(2) $R_{i} / D_{i-1}^{\prime}$ is reduced.

(3) $D_{i} / D_{i-1}^{\prime}$ is divisible. 
(4) $\kappa \leq\left|R_{i} / D_{i-1}^{\prime}\right| \leq \kappa^{\aleph_{0}}$.

(5) $D_{i}^{\prime} \leq \gamma_{i+2}(N)$.

Let $D_{0}=N$ and write $N / N^{\prime}$ as a direct sum of a divisible group $D_{1} / D_{0}^{\prime}$ and a reduced group $R_{1} / D_{0}^{\prime}$. Clearly $D_{0}^{\prime} \leq \gamma_{2}(N)$. If $B_{1} / D_{0}^{\prime}$ is a basic subgroup of $R_{1} / D_{0}^{\prime}$ then $B_{1}$ is a basic subgroup of $D_{0}$, so by $[14, \mathrm{XIV}]$

$$
\left|B_{1}\right|=\left|N / N^{\prime} N^{p}\right|=\kappa \text {. }
$$

It follows from [6, Corollary 34.4] that $\kappa \leq\left|R_{1} / D_{0}^{\prime}\right| \leq \kappa^{\aleph_{0}}$. Note in particular that (1) implies that $\left|N^{\prime}\right| \leq \kappa$.

Suppose that we have constructed $R_{i}$ and $D_{i}$, satisfying (1)-(5). Let $D_{i} / D_{i}^{\prime}$ $=R_{i+1} / D_{i}^{\prime} \oplus D_{i+1} / D_{i}^{\prime}$ with $D_{i+1} / D_{i}^{\prime}$ divisible and $R_{i+1} / D_{i}^{\prime}$ reduced. Let $B_{i+1} / D_{i}^{\prime}$ be a basic subgroup of $R_{i+1} / D_{i}^{\prime}$. Then $B_{i+1}$ is a basic subgroup of $D_{i}$, so

$$
\left|B_{i+1}\right|=\left|D_{i} / D_{i}^{\prime} D_{i}^{p}\right|=\left|D_{i} / D_{i}^{p}\right| \text {. }
$$

However, $D_{i}=D_{i}^{p} D_{i-1}^{\prime}$, so $D_{i} / D_{i}^{p} \cong D_{i-1}^{\prime} /\left(D_{i-1}^{\prime} \cap D_{i}^{p}\right)$. This has cardinality at most $\left|N^{\prime}\right|$, which is at most $\kappa$. Hence by [6, Corollary 34.4], $\kappa \leq\left|R_{i+1} / D_{i}^{\prime}\right| \leq$ $\kappa^{\aleph_{0}}$. Also $D_{i+1} / D_{i}^{\prime}$ is divisible and $D_{i+1} / \gamma_{3}\left(D_{i}\right)$ is abelian-by-divisible, so [14, XI] implies $D_{i+1} / \gamma_{3}\left(D_{i}\right)$ is abelian. Hence,

$$
D_{i+1}^{\prime} \leq \gamma_{3}\left(D_{i}\right) \leq \gamma_{3}\left(\gamma_{i+2} N\right) \leq \gamma_{i+3}(N),
$$

and the induction proceeds.

Since $D_{c-1}^{\prime} \leq \gamma_{c+1}(N)=1$, and since $N$ is reduced, $D_{c}=1$. Hence $D_{c-1}=$ $R_{c}$. It then follows that $\kappa \leq|N| \leq \kappa^{\aleph_{0}}$. This completes the proof.

The next lemma will prove to be repeatedly useful. For cardinal numbers $\mathfrak{m}, \mathfrak{n}$, let

$$
d(\mathfrak{m}, \mathfrak{n})= \begin{cases}\mathfrak{m} \mathfrak{n} & \text { if } \mathfrak{m} \text { is finite, } \\ \mathfrak{n}^{\mathfrak{m}} & \text { if } \mathfrak{m} \text { is infinite and } \mathfrak{n} \neq 1, \\ 2^{\mathfrak{m}} & \text { if } \mathfrak{m} \text { is infinite and } \mathfrak{n}=1\end{cases}
$$

4.3. Lemma. Suppose $G$ is a semicomplete nilpotent group. Let $r=r\left(Q / Q^{\prime} Q^{p}\right)$ and let $t=r_{p}(Z)$. Then $\left(Z_{2} / Z_{1}\right)_{p}$ contains a subgroup of rank $d(r, t)$.

Proof. Since $Q / Q^{\prime} Q^{p}$ is an epimorphic image of $Q_{\mathrm{ab}}$, there is a monomorphism

$$
\operatorname{Hom}\left(Q / Q^{\prime} Q^{p}, Z\right) \rightarrow \operatorname{Hom}\left(Q_{\mathrm{ab}}, Z\right) \cong Z_{2} / Z_{1} \text {. }
$$

Since $t=r(Z[p])$, we have

$$
\operatorname{Hom}\left(Q / Q^{\prime} Q^{p}, Z\right) \cong \operatorname{Hom}\left(\bigoplus_{r} C_{p}, Z\right) \cong \prod_{r} Z[p] \cong \prod_{r} \bigoplus_{t} C_{p},
$$

which has rank $d(r, t)$, as required.

Blackburn has shown that if $N$ is a nilpotent group of class $c$ and $p$ is a prime then there is a positive integer $f(p, c)$ such that, for every $n \geq f(p, c)$, every product of $p^{n}$ th powers of elements of $N$ is a $p^{n-f(p, c)}$ th power (see [13, Theorem 6.4]). Hence if $x \in N_{p}$ and $x \notin N_{p}^{p}$, then $x \notin N^{p^{1+f(p, c)}}$.

Proof of Theorem B. Suppose $\left|Q / Q^{\prime} Q^{p}\right|=\mathfrak{m}>\aleph_{0}$. By the remarks above and 4.1 we have

$$
\left|Q_{p} / Q_{p}^{p}\right| \leq\left|Q / Q^{p^{1+f(p, c)}}\right|=\left|Q / Q^{\prime} Q^{p}\right|
$$


It follows from 2.11 that $Q_{p}$ is reduced and 4.3 shows $Q_{p}$ is not finite. Hence $\left|Q_{p} / Q_{p}^{p}\right|=\kappa$ is infinite and 4.2 shows that $\kappa \leq\left|Q_{p}\right| \leq \kappa^{\aleph_{0}}$. However, by 4.3 again, $\left|Q_{p}\right| \geq 2^{\mathrm{m}}>2^{\aleph_{0}}$, so $\kappa \neq \aleph_{0}$. Thus $\left|Q_{p}\right|=\kappa=\left|Q_{p} / Q_{p}^{p}\right|$. This is a contradiction since $\kappa \leq \mathfrak{m}$.

\section{THE PROOF OF THEOREM A}

We note first that if $Q_{\mathrm{ab}}$ is $p$-divisible then $\left(Z_{2} / Z_{1}\right)[p] \cong \operatorname{Hom}\left(Q_{\mathrm{ab}}, Z\right)[p]=$ 0 by $[6, \S 43(\mathrm{E})]$, contradicting the fact that $p \in \pi(Q)$. Hence $Q / Q^{\prime} Q^{p} \neq 0$. Our next result is almost immediate from what we have done already.

5.1. Proposition. Suppose that $G$ is a semicomplete nilpotent group. If $Q_{p}$ is finite then $G_{p}$ is finite.

Proof. It follows from 4.3 and the fact that $Q_{p}$ is finite that $r\left(Z_{p}\right)$ is finite. Hence $Z_{p}$ is finite, since it is reduced. Thus $G_{p}$ is finite.

We now have

Proof of Theorem A. (a) Assume that $Q / Q^{\prime} Q^{p}$ is finite. Then by 4.2(i) it follows that $Q / Q^{p}$ is finite. As in the proof of Theorem $\mathrm{B}, Q_{p} / Q_{p}^{p}$ is also finite, so, by 4.2 (i) again, $Q_{p}$ is finite and the result now follows by 5.1 .

(b) Suppose that $Q_{p}$ is countable. By 5.1, we may assume that $Q_{p}$ is infinite. Let $\mathfrak{m}=\left|Q / Q^{\prime} Q^{p}\right|$. By 4.3 , if $\mathfrak{m}$ is infinite then $\left|Q_{p}\right| \geq 2^{\mathfrak{m}}$, a contradiction. Hence $\mathfrak{m}$ is finite and the result follows from part (a).

(c) By part (a) and Theorem B we need only consider the case $\left|Q / Q^{\prime} Q^{p}\right|=\aleph_{0}$. Let the exponent of $Q_{p}$ be $p^{n}$. Then by 4.1 and the remarks preceding the proof of Theorem B, $\left|Q_{p}\right|=\left|Q_{p} / Q_{p}^{p^{n}}\right| \leq\left|Q / Q^{\prime} Q^{p}\right|=\aleph_{0}$. The result now follows from part (b).

(d) Suppose that $G$ is nilpotent of class 2 . In this case $Q \cong$ Aut $G \cong$ $\operatorname{Hom}(Q, Z)$, and by the preceding results we only need consider the situation when $\left|Q_{p}\right|>\aleph_{0}$ but $|Q / p Q|=\aleph_{0}$. (We revert here to additive notation for $Q$.) As before we have $\left|Q_{p} / p Q_{p}\right| \leq|Q / p Q|=\aleph_{0}$. Also $\aleph_{0}<\left|Q_{p}\right| \leq 2^{\aleph_{0}}$. Since $Q_{p}$ is reduced, it follows from [6, Corollary 27.3] that $Q=C_{p^{n}} \oplus X$, for some $n$ and some $X$. Suppose that $Z_{p}$ has infinite rank $t$. Then

$$
\begin{aligned}
Q & \cong \operatorname{Hom}(Q, Z)=\operatorname{Hom}\left(C_{p^{n}}, Z\right) \oplus \operatorname{Hom}(X, Z) \\
& \cong Z\left[p^{n}\right] \oplus K \cong \bigoplus_{\mathfrak{t}} C_{p^{n(i)}} \oplus K
\end{aligned}
$$

where $K=\operatorname{Hom}(X, Z)$ and $n(i) \leq n$ for all $i$. Hence

$$
\begin{aligned}
Q & \cong \operatorname{Hom}(Q, Z) \cong \operatorname{Hom}\left(\bigoplus_{\mathfrak{t}} C_{p}{ }^{n(i)}, Z\right) \oplus \operatorname{Hom}(K, Z) \\
& \cong \prod_{\mathfrak{t}} Z\left[p^{n(i)}\right] \oplus \operatorname{Hom}(K, Z) .
\end{aligned}
$$

Now $\prod_{\mathrm{t}} Z\left[p^{n(i)}\right]$ has exponent bounded by $p^{n}$ and if we set $L=\operatorname{Hom}(K, Z)$ then we have

$$
Q=\bigoplus_{2^{\ddagger}} C_{p}^{m(i)} \oplus L, \quad m(i) \leq n \text { for all } i .
$$


Substituting again into $Q \cong \operatorname{Hom}(Q, Z)$ we obtain a $p$-subgroup of $Q$ of cardinality $2^{2^{t}}$, contradicting the fact that $\left|Q_{p}\right| \leq 2^{\aleph_{0}}$. Thus $\mathfrak{t}$ is finite and so $Z_{p}$ is finite since it is reduced. Hence $Q_{p}$ is of bounded exponent and the desired result follows from part (c). This completes the proof of Theorem A.

\section{ACKNOWLEDGMENT}

It is a pleasure to record our thanks to Tom Fournelle who suggested the problem with which this paper is concerned. We would also like to thank the referee for suggesting several improvements to the paper.

\section{REFERENCES}

1. T. A. Fournelle, Outer automorphisms of nilpotent groups, Bull. London Math. Soc. 13 (1981), 129-132.

2. _ Nilpotent groups without periodic automorphisms, Bull. London Math. Soc. 15 (1983), 590-595.

3. _ Torsion in semicomplete nilpotent groups, Math. Proc. Cambridge Philos. Soc. 94 (1983), 191-202.

4. __ Automorphisms of nilpotent groups of class two with small rank, J. Austral. Math. Soc. Ser. A 39 (1985), 121-131.

5. S. Franciosi and F. deGiovanni, A note on groups with countable automorphism group, Arch. Math. 47 (1986), 12-16.

6. L. Fuchs, Infinite abelian groups, Academic Press, New York, 1973.

7. W. Gaschütz, Kohomologische Trivialität und äussere Automorphismen von p-Gruppen, Math. Z. 88 (1965), 432-433.

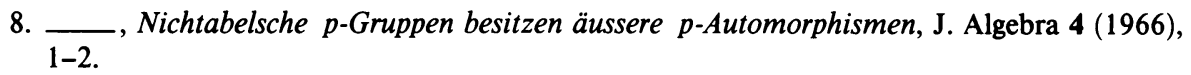

9. H. Heineken, Automorphism groups of torsionfree nilpotent groups of class 2, Sympos. Math. 17 (1976), 235-250.

10. D. J. S. Robinson, Outer automorphisms of torsionfree nilpotent groups, preprint.

11. $\longrightarrow$ Finiteness conditions and generalized soluble groups (2 vols.), SpringerVerlag, New York, 1972.

12. __ Infinite torsion groups as automorphism groups, Quart. J. Math. Oxford (2) 30 (1979), 351-364.

13. R. B. Warfield, Nilpotent groups, Lecture Notes in Math., vol. 513, Springer-Verlag, New York, 1972.

14. A. E. Zalesskii, A nilpotent p-group possesses an outer automorphism, Dokl. Akad. Nauk. SSSR 196 (1971), 751-754; English transl., Soviet Math. Dokl. 12 (1971), 227-230.

15. __ An example of a torsionfree nilpotent group having no outer automorphisms, Mat. Zametki 11 (1972), 21-26; English transl., Math. Notes 11 (1972), 16-19. 0350

Department of Mathematics, University of Alabama, Tuscaloosa, Alabama 35487 -

E-mail address: mdixon at ualvm.bitnet.

Department of Mathematics and Science, Troy State University at Dothan, Dothan, Alabama 36304

Current address: Division of Math/Science/Engineering, Westark Community College, Ft. Smith, Arizona 72913 